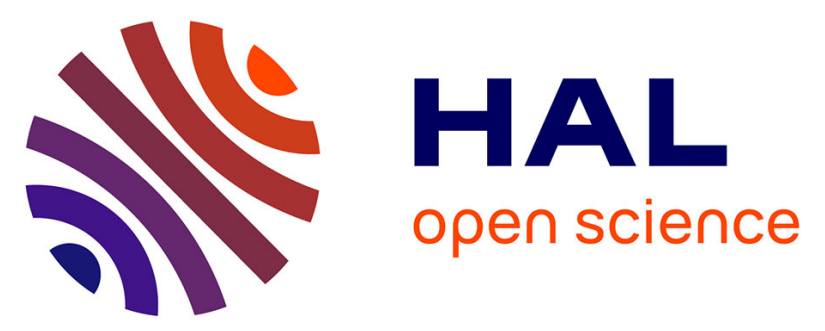

\title{
Making Local Algorithms Wait-Free: the Case of Ring Coloring
}

\author{
Armando Castañeda, Carole Delporte-Gallet, Hugues Fauconnier, Sergio
} Rajsbaum, Michel Raynal

\section{- To cite this version:}

Armando Castañeda, Carole Delporte-Gallet, Hugues Fauconnier, Sergio Rajsbaum, Michel Raynal. Making Local Algorithms Wait-Free: the Case of Ring Coloring. Theory of Computing Systems, 2019, pp.344-365. 10.1007/s00224-017-9772-y . hal-01672723

\section{HAL Id: hal-01672723 \\ https://hal.science/hal-01672723}

Submitted on 3 Jan 2018

HAL is a multi-disciplinary open access archive for the deposit and dissemination of scientific research documents, whether they are published or not. The documents may come from teaching and research institutions in France or abroad, or from public or private research centers.
L'archive ouverte pluridisciplinaire HAL, est destinée au dépôt et à la diffusion de documents scientifiques de niveau recherche, publiés ou non, émanant des établissements d'enseignement et de recherche français ou étrangers, des laboratoires publics ou privés. 


\title{
Making Local Algorithms Wait-Free: The Case of Ring Coloring
}

\author{
Armando Castañeda • Carole Delporte • \\ Hugues Fauconnier • Sergio Rajsbaum • \\ Michel Raynal
}

Received: date / Accepted: date

\begin{abstract}
When considering distributed computing, reliable message-passing synchronous systems on the one side, and asynchronous failure-prone sharedmemory systems on the other side, remain two quite independently studied ends of the reliability/asynchrony spectrum. The concept of locality of a computation is central to the first one, while the concept of wait-freedom is central to the second one. The paper proposes a new $\mathcal{D E C O U P} \mathcal{L} \mathcal{E} D$ model in an attempt to reconcile these two worlds. It consists of a synchronous and reliable communication graph of $n$ nodes, and on top a set of asynchronous crash-prone processes, each attached to a communication node.

To illustrate the $\mathcal{D E C O U} \mathcal{P} \mathcal{L} \mathcal{E} D$ model, the paper presents an asynchronous 3 -coloring algorithm for the processes of a ring. From the processes point of view, the algorithm is wait-free. From a locality point of view, each process uses information only from processes at distance $O\left(\log ^{*} n\right)$ from it. This local wait-free algorithm is based on an extension of the classical Cole and Vishkin's vertex coloring algorithm in which the processes are not required to start simultaneously.
\end{abstract}

Keywords Synchronous distributed computing · Cole and Vishkin's coloring algorithm · Locality of a computation · Message-passing · Process crash failure · Synchronous communication · Vertex coloring problem · Waitfreedom

\footnotetext{
Armando Castañeda · Sergio Rajsbaum

Instituto de Matemáticas, UNAM, Mexico City, México

E-mail: armando.castanedar@im.unam.mx, rajsbaum@matem.unam.mx

Carole Delporte $\cdot$ Hugues Fauconnier

IRIF, Université Paris Diderot, Paris, France

E-mail: \{carole.delporte, hugues.fauconnier\}@liafa.univ-paris-diderot.fr

Michel Raynal

IUF \& IRISA (Université de Rennes), Rennes, France

E-mail: michel.raynal@irisa.fr
} 


\section{Introduction}

Locality in synchronous distributed computing. The standard synchronous message passing model (e.g. see [21,22]) consists of a graph, whose vertices represent computational processes, each with a unique ID, and whose edges represent bidirectional communication links. In each synchronous round, a process sends messages to its neighbors, then receives messages from them, and finally performs arbitrary computations. Failures are not considered: each message is received in the same round in which it was sent, and processes do not fail. The time complexity of a distributed algorithm in this model is the maximum number of rounds any process requires to terminate.

In sequential computing only the most trivial tasks can be solved in constant time. In contrast, there are many synchronous distributed algorithms that run in a number of rounds $d$ which is constant (or nearly constant), independently of the number of vertices of the graph [25]. In such an algorithm, a process is able to collect information from others at most $d$ links away, and hence we can think of the algorithm as a function that maps the $d$-neighborhood of a node to a local output, for each node. In synchronous distributed computing the focus is on locality, or to what extent a global property about the graph can be obtained from locally available data [18].

The study of the $\mathcal{L O C} \mathcal{A} \mathcal{L}$ synchronous model was initiated at the very early days of distributed computing [21], with problems such as coloring the vertices of a ring with 3 colors. This is a problem that depends globally on the ring, yet it can be solved locally. Cole and Vishkin [8] designed an algorithm that finds a 3 -coloring of the vertices of a ring in $O\left(\log ^{*} n\right)$ rounds. Soon after, Linial proved that $\Omega\left(\log ^{*} n\right)$ rounds are needed for 3 -coloring a ring. For general graphs, it is known that $(\Delta+1)$-coloring can be done in time $O\left(\Delta+\log ^{*} n\right)[7]$, and only recently it was shown that it can be done in time $o(\Delta)+O\left(\log ^{*} n\right)[4,10]$, where $\Delta$ is the largest degree in the graph. Developments on what can or cannot be locally computed can be found in many papers (e.g., $[5,17,18,20]$ to cite a few; more references can be found in the survey [25]). This part of distributed computing is mainly complexity-oriented $[12,21]$, as every decidable problem can be solved in $D$ rounds, where $D$ equal to the diameter of the graph.

Fault-tolerance in asynchronous distributed computing. At the same time that the $\mathcal{L O C} \mathcal{A} \mathcal{L}$ model began to be studied, ignoring asynchrony and failures, an orthogonal branch of distributed computing was beginning to focus on faulttolerance, and disregarding the communication network topology [9, 15]. In an asynchronous crash-prone distributed computing model [23,24], (i) there are communication links between every pair of processes, (ii) there are no bounds on message transfer delays and each process runs at its own arbitrary speed, which can vary along with time, and (iii) processes can fail by crashing. In this area, consensus is a fundamental problem, because, roughly speaking, it allows processes to agree on a function of their inputs, which can then be used by each process to individually perform a consistent computation. However, it was proved early on that there is no deterministic distributed asynchronous 
message-passing consensus algorithm even if only one process may crash [9]. Hence, computability questions are central in this part of distributed computing. Given assumptions about how many processes may fail, how severe the failures can be, and other assumptions about communication, one tries to identify the distributed problems that are solvable in a specific model.

Reliable message-passing synchronous systems and asynchronous failureprone systems remain two quite independently studied poles of distributed computing.

Aim and content of the paper. In a distributed system failures and asynchrony are rarely coming from the hardware, but much more often from the software. Hence, it is natural to consider a model composed of two distinct layers, with distinct reliability and synchrony features, namely:

- A synchronous and reliable communication graph $G$ with $n$ nodes, and

- $n$ asynchronous crash-prone processes, each one attached to a distinct node.

At each vertex of $G$ there are two components: a failure-free synchronous node in charge of communicating with the nodes of its neighbors, and a failure-prone asynchronous process in charge of performing the actual computation. Notice that, in contrast to the $\mathcal{L O C} \mathcal{A} \mathcal{L}$ model, in the $\mathcal{D E C O U} \mathcal{P} \mathcal{L} \mathcal{D}$ model after $d$ rounds of communication, a process can collect the local inputs of only a subgraph of its $d$-neighborhood, since processes can start at distinct times and run at different speeds. Thus, the new model is in principle more challenging than the $\mathcal{L O C} \mathcal{A L}$ model.

To illustrate the $\mathcal{D E C O U P} \mathcal{L} \mathcal{E} \mathcal{D}$ model approach, the paper considers a fundamental problem of failure-free synchronous distributed computing. It presents a 3-coloring algorithm for a ring, denoted WLC (for Wait-free Local Coloring), suited to the $\mathcal{D E C O U P} \mathcal{L} \mathcal{E D}$ model. This algorithm is based on the time-optimal Cole and Vishkin's vertex coloring algorithm, which is denoted CV86 in the following $[8]^{1}$. The CV86 algorithm runs in $\log ^{*} n+3$ rounds ${ }^{2}$ while the new algorithm runs in $\log ^{*} n+6$ rounds. From the processes point of view, the algorithm is fully asynchronous, wait-free, i.e., a process never waits for an event in another process. Yet the algorithm is local, in the sense that each process uses information only from processes at distance $O\left(\log ^{*} n\right)$ from it. Moreover, this amount of information is asymptotically optimal due to Linial's lower bound [18] and because in the absence of failures and asynchrony, the $\mathcal{D E C O U P} \mathcal{L} \mathcal{E D}$ model boils down to the $\mathcal{L O C} \mathcal{A} \mathcal{L}$ model.

The WLC algorithm for the $\mathcal{D E C O U P} \mathcal{L} \mathcal{E D}$ model is built in two stages. First an extension of CV86 is presented that may be interesting in itself. This extension, denoted AST-CV, is an implementation of CV86 in a synchronous system where reliable processes need not start at the very same round. The

\footnotetext{
1 CV86 was designed for trees in the PRAM model. It can be easily adapted to failure-free message-passing synchronous systems, for a ring, or a chain of processes.

2 Assuming $n \geq 2, \log ^{*} n$ is the number of times the function " $\log _{2}$ " needs to be applied in the invocation $\log _{2}\left(\log _{2}\left(\log _{2} \ldots\left(\log _{2} n\right) \ldots.\right)\right)$ to obtain value 1 . Let us remember that $\log ^{*}$ (approx. number of atoms in the universe) $=5$.
} 
main idea of the first stage is to run CV86 within each segment of the ring that happens to wake up at precisely the same time. Then, adjacent endpoints of such segments fix their colors by giving priority to the segment that began earlier. Somewhat surprisingly this approach works even when all segments happen to consist of a single process. In the second stage it is shown how to derive the wait-free algorithm WLC from AST-CV. When a process starts (asynchronously with respect to other processes), it obtains information on the "current state" of the processes at distance at most $O\left(\log ^{*} n\right)$ from it; then, using the information obtained, the process executes alone a purely local simulation of AST-CV, at the end of which it obtains its final color.

The new algorithm shows how it is possible to extend the scope of a synchronous failure-free algorithm to run on asynchronous and crash-prone processes, without losing its fundamental locality properties, and at the cost of only a small constant number of rounds. Up to the best of our knowledge this is the first time the design of fault-tolerant asynchronous algorithms on top of a synchronous communication network is considered from the locality perspective. However this is certainly not the first work that relates synchronous and asynchronous systems, a few examples follow. From very early on the performance of asynchronous processes with access to a global clock has been considered [1]. The performance of wait-free algorithms running on top of partially synchronous, fully-connected systems has been of interest for some time, e.g. $[11,16]$. The opposite problem, of running a synchronous algorithm in an asynchronous (failure-free) network was introduced in [2], and there are extensions even to the case where links are assumed to crash and recover dynamically [3]. In globally asynchronous locally-synchronous (GALS) design for microprocessor networks, the system is partitioned into synchronous blocks of logic which communicate with each other asynchronously [19]. An example of a reliable network infrastructure is provided by the highly popular Synchronous Optical Networking (SONET), which provides synchronous transport signals for fiber-optic based transmissions on top of which asynchronous algorithms may be deployed.

Roadmap The paper is composed of 6 sections. Section 2 presents the first contribution, namely the two-component-based computation $\mathcal{D E C O U P \mathcal { L E D }}$ model. The WLC algorithm is built incrementally. Section 3 presents first the distributed graph coloring problem and a version of CV86 tailored for a ring, which is the starting point of WLC. Then, Section 4 presents an extension of CV86 (denoted AST-CV) suited to synchronous reliable systems, which does not require the processes to start participating in the algorithm at the very same time. Section 5 shows that a local wait-free algorithm (WLC) can be obtained in two stages: after it started (asynchronously with respect to the other processes), a process executes first a communication stage during which it obtains information on the "current state" of the processes at distance at most $O\left(\log ^{*} n\right)$ from it; then, using the information previously obtained, it executes a second stage, which is a purely local simulation of AST-CV, at the end of which it obtains its final color. Finally, Section 6 concludes the paper. 


\section{The Two-Component-Based Model}

Here the $\mathcal{D E C O U} \mathcal{P} \mathcal{L} \mathcal{E} D$ model is presented, where asynchronous crash-prone processes running a wait-free algorithm are mounted on top of a reliable, synchronous network.

Communication component. The communication component is modelled by a connected graph $G$ of $n$ vertices. Its vertices represent nodes, $n d_{1}, \ldots, n d_{n}$. Each node $n d_{i}$ is a communication device connected with two types of entities. It is connected with its neighbor nodes in $G$, and to its local process $p_{i}$, in charge of running the wait-free algorithm. A node is connected to each of these entities through an input port and an output port. Moreover, a node $n d_{i}$ is a device in charge only of transmitting and receiving messages (the actual computation of the wait-free algorithm is performed by the process $p_{i}$ ).

Each edge of $G$ represents a reliable communication link, which does not corrupt, lose, create, nor duplicate messages. Similarly, nodes do not fail in any way. The communication component is synchronous. All its entities and message exchanges proceed in a lock-step manner. More precisely, there is a global clock which governs the progress of the communication component: at every clock tick ${ }^{3}$, each node $n d_{i}$ reads its input ports (from its neighbor nodes, and from its process $p_{i}$ ), composes a message from what it has read, and sends this message on all its output ports (to its neighbor nodes, and to $\left.p_{i}\right)$. Every message is received in the same clock tick as the one in which it was sent. Recall that the communication component is always active: at every clock tick, each node $n d_{i}$ sends and receives messages, independently of the behaviour of its associated process $p_{i}$.

Computing component. Each communication node $n d_{i}$ has an associated sequential process $p_{i}$. A process $p_{i}$ can communicate only with its node $n d_{i}$. A process is asynchronous, which means that it proceeds at its own speed, which can vary along with time, and is independent of the speed of other processes. Moreover, processes may crash, and when a process crashes it never recovers. As processes are asynchronous, they can wake up at arbitrary times to participate in an algorithm. Therefore, as the communication component is reliable, when a process wakes up, it may find messages from its input port waiting to be read, which were sent by its neighbors that started the algorithm before it, as described below.

Interaction between the components. The input and output ports connecting a process $p_{i}$ with its node $n d_{i}$ have two buffers (in our algorithms they are bounded). The one denoted out $t_{i}$ is from $p_{i}$ to $n d_{i}$, while the one denoted $i n_{i}$ is from $n d_{i}$ to $p_{i}$, initially empty. When a process starts, it writes in out $t_{i}$ some

\footnotetext{
3 We use the "time" and "clock tick" terminology for the communication component, to prevent confusion with the "round" terminology used in the description of the CV86 and AST-CV algorithms.
} 


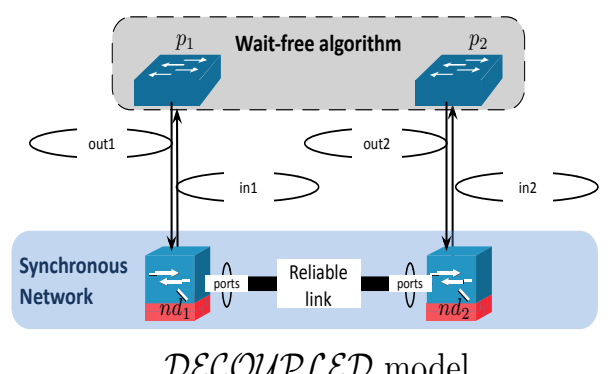

value, which may depend on the problem being solved. At every communication step, node $n d_{i}$ first receives a message from each of its neighbors, and

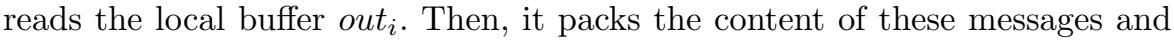
the current value of out $_{i}$ into a single message, sends it to its neighbors, and writes it in $i n_{i}$. Thus, due to asynchrony, it could be the case that the first time $p_{i}$ reads from $i n_{i}$, it sees messages from processes that started before.

The global ticks of the communication component govern when each communication step happens. In addition, each tick is associated to a global time. Given a process $p_{i}, t s_{i}$ is the global time at which $p_{i}$ wakes up and starts executing. Thanks to the underlying messages exchanged by the communication nodes at every clock tick (communication step), a process $p_{i}$ which started participating in the algorithm can know (a) which of its neighbors (until some predefined distance $D$ ) started the algorithm, and (b) at which time they started $^{4}$. More precisely, considering a process $p_{i}$ that starts at time $s t_{i}$, after $D$ time units, $p_{i}$ can have information from processes in the graph at distance up to $D$ from it.

Initial knowledge. Each of the $n$ pairs made up of a communication node $\left(n d_{i}\right)$ and a process $\left(p_{i}\right)$ has a unique identity $i d_{i}$. It is assumed that each identity can be encoded in $\log n$ bits. Initially, a process knows its identity, the value of $n$, and possibly the graph $G$. Moreover, while a process knows that no two processes have the same identity, it does not know the identities of the other processes.

Power of the model. The $\mathcal{D E C O U P} \mathcal{L} \mathcal{E} \mathcal{D}$ model behaves exactly like the $\mathcal{L O C} \mathcal{A} \mathcal{L}$ model, in the absence of failures and presence of synchrony: all processes run in lock-step manner until decisions are made. Thus, if there is an algorithm solving a given problem in $\mathcal{D E C O U P} \mathcal{L} \mathcal{E} \mathcal{D}$, then one can easily obtain an algorithm solving the corresponding problem in $\mathcal{L O C} \mathcal{A L}$. The rest of the paper presents WLC, a 3-vertex coloring algorithm for a ring, showing that, in principle, the other direction is possible as well.

\footnotetext{
4 The assumption that processes know the global time is made only to simplify the description of our algorithms. All that a process $p_{i}$ needs to know is the relative order of wake up with respect to its neighbors, which can be deduced from the content of the buffers at wake up time $s t_{i}$.
} 


\section{Distributed Graph Coloring and a Look at Cole and Vishkin's Algorithm}

In the 3-coloring problem, each vertex of a graph is assigned a color from a set of three possible colors, in such a way that no two adjacent vertices have the same color. In sequential computing, deciding if a graph can be 3-colored is a famous NP-complete problem [13].

\subsection{Graph coloring}

In the context of synchronous systems, there is an $\Omega\left(\log ^{*} n\right)$ rounds lower bound on the number communication rounds needed to 3-color the nodes of a ring [18], and the CV86 algorithm solves the problem in $\log ^{*} n+3$ rounds [8]. A monograph entirely devoted to distributed graph coloring can be found in [6].

The structure of Cole and Vishkin's algorithm. This algorithm assumes that the underlying bi-directional communication graph has a logical orientation, such that each process has at most a single predecessor. It assumes that the processes have distinct identities, each consisting of $O(\log n)$ bits. The algorithm can be decomposed in two phases.

- Phase 1. From $n$ colors to six colors. An original and clever bit-level technique is first used (see below), which allows the processes to be properly colored with six colors. Starting with colors encoded with $\log n$ bits (node identities), a sequence of synchronous communication steps is executed, such that in each step a process computes a new proper color whose size in bits is exponentially smaller than the previous one. This is repeated until attaining at most six colors, which requires $\log ^{*} n$ communication rounds.

- Phase 2. From six colors to three colors. The algorithm uses then a simple reduction technique to reduce the number of colors from six to three. This requires three additional rounds (each one eliminating a color).

Features of CV86. Those are the following: it is local, it's time complexity is $\log ^{*} n+3^{5}$, time asymptotically optimal [18], and deterministic. Combining the locality and determinism properties, it follows that the final color of a process depends only on the $\log ^{*} n+3$ identities of the processes on its predecessor path.

\subsection{A version of Cole and Vishkin's algorithm suited to a ring}

A version of CV86 suited to a ring is given in Figure 1. The two neighbors of a process $p_{i}$ are denoted pred $_{i}$ and next $t_{i}$. The local variable color ${ }_{i}$ contains

\footnotetext{
5 Usually, local refers to time complexity $O(1)$. Here we adopt the more broad definition of time complexity $o(D)$, where $D$ is the diameter of the graph.
} 
initially the identity of $p_{i}$, using $\log n$ bits. Let $m=\lceil\log n\rceil-1$. The initial value of color $_{i}$ is a sequence of $(m+1)$ bits $b_{m}, b_{m-1}, \cdots, b_{1}, b_{0}$, and no two processes have the same initial sequence of bits. We say that " $b_{y}$ is at position $y$ ", i.e., the position of a bit in a color is defined by starting from position 0 and going from right to left.

Underlying principle. The aim is, from round to round, to compress as much as possible the size of the colors of the processes, while keeping invariant the property that no two neighbors have the same color. Basically, a process compares its current color with the one of its predecessor, to define its new color (using the logical orientation of the ring).

The two issues that have then to be solved are (i) how to compare current colors and how to compute a new shorter color (while maintaining adjacent processes with different colors), and (ii) how many iterations have to be executed to get to at most three colors.

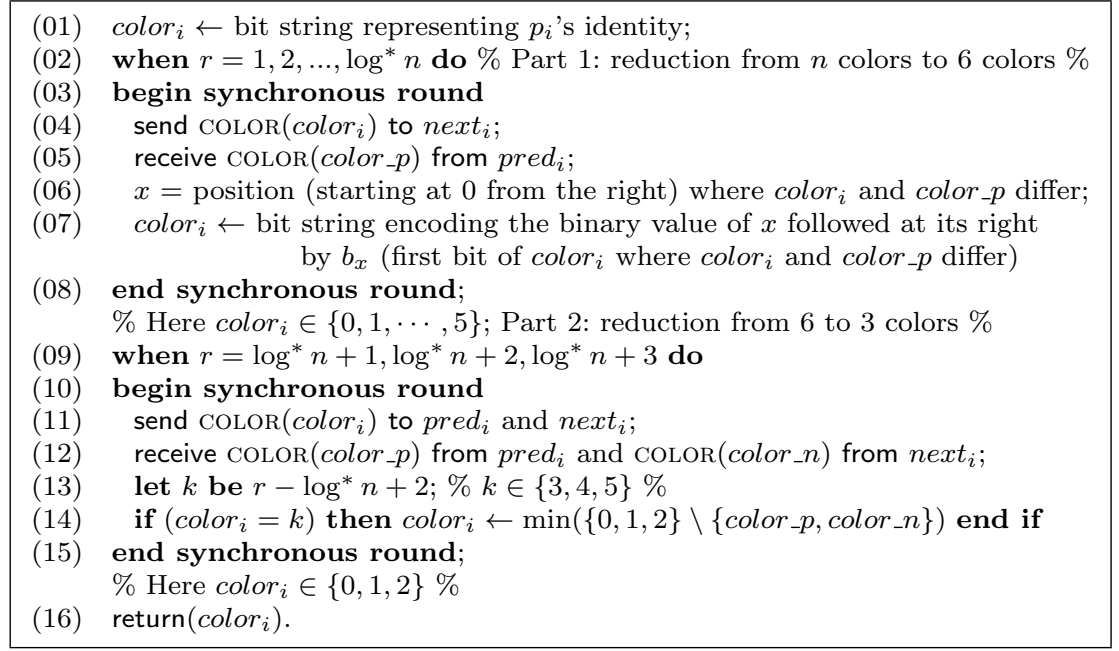

Fig. 1 Cole and Vishkin's synchronous algorithm for a ring (code for $p_{i}$ )

Description of the algorithm. Let $r$ denote the current round number. Initialized to 1 , it takes then the successive values 2 , 3 , etc. It is a global variable provided by the synchronous system, which can be read by all processes. Each process $p_{i}$ first defines its current color as the bit string representing its identity (line 01). As already indicated, it is assumed that each identity can be coded in $\log n$ bits. Then $p_{i}$ executes synchronous rounds until it obtains its final color (line 16). The total number of rounds that are executed is $\log ^{*} n+3$, which decompose into two parts.

The first $\log ^{*} n$ rounds (lines 03-08) allow each process $p_{i}$ to compute a color in the set $\{0,1, \cdots, 5\}$. Considering a round $r$, let $k$ be an upper bound on the 
number of different colors at the beginning of round $r$, and $m$ be the smallest integer such that $k \leq 2^{m}$. Hence, at round $r$, the color of a process is coded on $m$ bits. After a send/receive communication step (lines 04-05), a process $p_{i}$ compares its color with the one it has received from its predecessor (color_p), and computes (starting at 0 from the right), the rightmost bit position $x$ where they differ (line 06). Assuming for example that $k=2^{8}$ (hence $m=8$ ), let color $_{i}=10011001$ and color_p $=11011101$; we have then $x=2$. Then (line 07$), p_{i}$ defines its new color as the bit sequence whose prefix is the binary encoding of $x$ in $\log m$ bits (010 in our example) and suffix is the first bit of its current color where both colors differ, namely $b_{x}\left(b_{x}=b_{2}=0\right.$ in the example). Hence, its new color is $010 b_{x}=0100$.

Consider two neighbor processes during a round $r$. If they have the same value for $x$, due to the bit suffix they use to obtain their new color, they necessarily obtain different new colors. If they have different values for $x$, they trivially have different new colors. It is easy to see (from the computation of the position $x$-which defines the prefix of the new color-, and the value of the bit $b_{x}$-which defines the suffix of the new color-), that the round $r$ reduces the number of colors from $k$ to at most $2\lceil\log k\rceil \leq 2 \mathrm{~m}$. It is shown in [8] that, after at most $\log ^{*} n$ rounds, the binary encoding of a color requires only three bits, where the suffix $b_{x}$ is 0 or 1 , and the prefix is 00,10 , or 01 . Hence, only six color values are possible: 000, 100, 010, 001, 101, and 011 .

The second part of the algorithm consists of three additional rounds, each round eliminating one of the colors in $\{3,4,5\}$ (lines 10-15). Each process first exchanges its color with its two neighbors. Due to the previous $\log ^{*} n$ rounds, these three colors are different. Hence, if its color is $3, p_{i}$ selects any color in $\{0,1,2\}$ not owned by its neighbors. This is then repeated twice to eliminate the colors 4 and 5 .

Proofs of the algorithm correctness and its time complexity can be found in $[8]$.

From a ring to a chain. A chain is a sequence where each vertex appears at most once (a ring that has been cut). Hence, each non-singleton chain has two processes that define its ends.

Cole-Vishkin's algorithm described in Figure 1 is modified as follows to work on a process chain. At line 05 , the process that has no predecessor cannot compare its current color with another color. It simply does as if it has a (fictitious) predecessor whose color is different from its initial color, and executes normally the algorithm. As an example, if $p_{i}$ (whose initial color is 100101) is the process without predecessor, it considers a fictitious predecessor whose color is the same as its color except for its first bit (starting from the right), i.e., the color 100100). It follows from the algorithm that after the first round, $p_{i}$ obtains the color 01 (which will never change thereafter as the fictitious predecesor has the same color in every round).

Finally, at line 12, an end process defines the color of its "missing neighbor" as being the "no-color" denoted -1 . 


\section{Extending Cole and Vishkin's Algorithm to Asynchronous Starting Times}

This section presents an extension of CV86 for synchronous systems, where reliable processes may start at different rounds.

\subsection{Asynchronous starting times and unit-segment}

Asynchronous starting times. Let $s_{i}$ denote the round number at which process $p_{i}$ wakes up and starts participating in the algorithm. A process may start at any of the discrete time points defined by beginning of rounds, and then runs synchronously.

Notion of a unit-segment. A unit-segment is a maximal sequence of consecutive processes in the ring, $p_{a}, p_{\text {next }_{a}}, \cdots, p_{\text {pred }_{z}}, p_{z}$, that start the algorithm in the same round.

A unit-segment is identified by a starting time (round number), and any two contiguous unit-segments are necessarily associated with distinct starting times. It follows that, from an omniscient observer's point of view, and at any time, the ring can be decomposed into a set of unit-segments, some of these unit-segments being contiguous, while others are separated by processes that have not yet started (or will never start, due to an initial crash). In the particular case where all processes start simultaneously, the ring is composed of a single unit-segment, and if all start at different times, the ring is composed of $n$ unit-segments.

\subsection{A coloring algorithm with asynchronous starting times}

This section presents the local algorithm AST-CV, which allows processes to start at different times. Each process executes $\Delta=\log ^{*} n+6$ rounds. The algorithm is decomposed into four parts.

Starting round of the algorithm. The underlying synchronous system defines the first round $(r=1)$ as being the round at which the first process(es) starts the algorithm. Hence, when such a process $p_{i}$ starts the algorithm, we have $s t_{i}=1$. Then, the progress of $r$ is managed by the system synchrony.

Part 1 and Part 2. These parts are described in Figure 2. Considering a unitsegment (identified by a starting time $s t$ ) they are a simple adaptation of CV86, which considers the behavior of any process $p_{i}$ belonging to this unit-segment.

A process $p_{i}$ executes first $\log ^{*} n$ synchronous rounds. During each round, it sends its current color to its neighbors, and receives their current colors. msg_pred $=\perp$ if there is no message from pred $_{i}$ (line 04). 
init: color $_{i}$ : bit string initialized to $p_{i}$ 's identity; $s t_{i}$ : starting round of $p_{i}$; when $p_{i}$ starts, there are three cases for each of its neighbors pred $_{i}$ and next $t_{i}$ :

(a) it already started the algorithm;

(b) it starts the algorithm at the very same round;

(c) it will start the algorithm at a later round.

In the first case, the messages sent in previous rounds by the corresponding neighbor are in $p_{i}$ 's input buffer, and can be consequently read by $p_{i}$.

In the last case, to simplify the presentation, we consider that $p_{i}$ receives a dummy message.

fict_pred $_{i}$ : fictitious process whose identity is the same as $p_{i}$ 's identity except for its first bit (starting from the right); used as predecessor in case $p_{i}$ discovers it is a left end of a unit-segment.

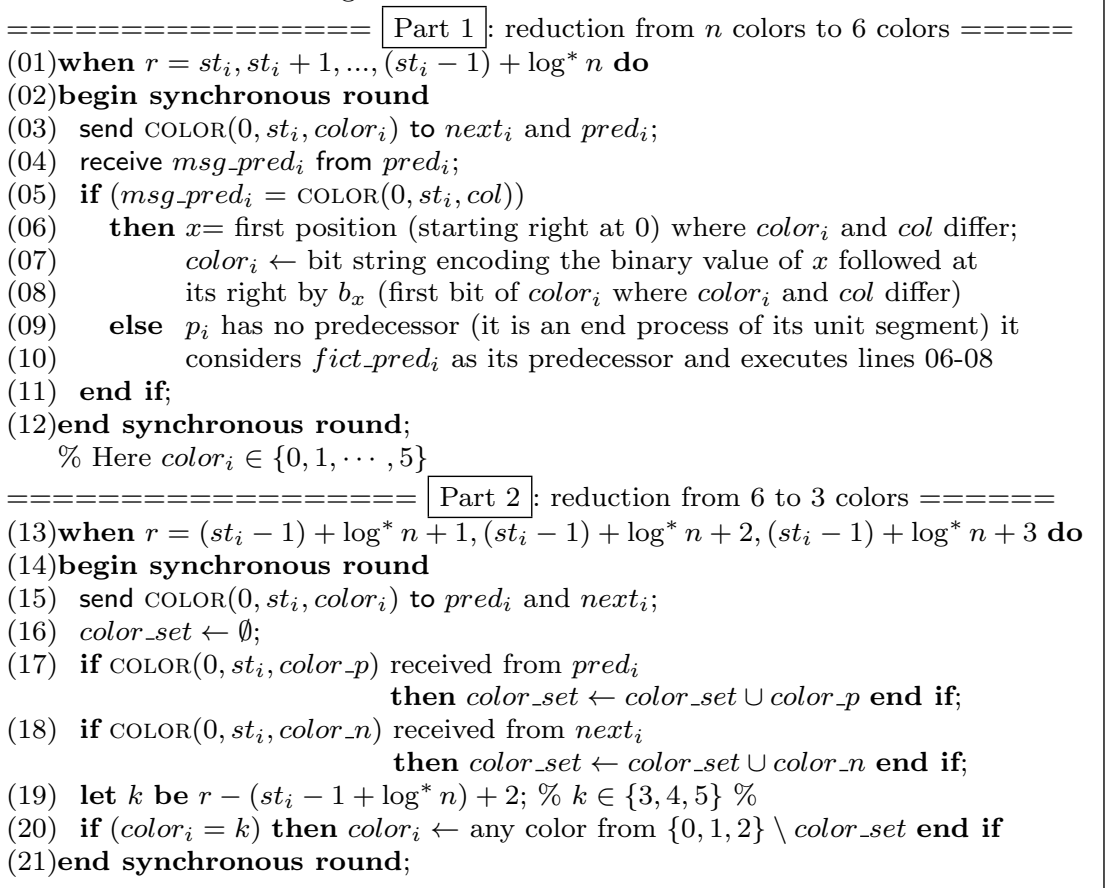

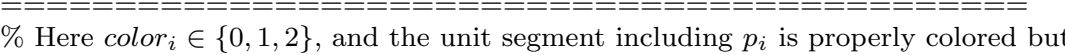
$\%$ two end processes of two consecutive unit segments may have the same color

Fig. 2 Initialization, Part 1, and Part 2, of AST-CV (code for $p_{i}$ )

In line $05, p_{i}$ can tell if its predecessor belongs to the same unit-segment from the $s t$ value received. If so, $p_{i}$ executes CV86. If its predecessor belongs to a different unit-segment or has not yet started the algorithm, $p_{i}$ considers a fictitious predecessor whose identity is the same as its own identity, except for the first bit, starting from the right (see the last paragraph of Section 3.2). Lines 06-10 constitute the core of CV86, which exponentially fast reduces the bit size representation of color $_{i}$ at every round, to end up with a color in the set $\{0,1, \cdots, 5\}$ after $\log ^{*} n$ rounds. 
Part 2 of AST-CV (lines 13-21) is the same as the part in CV86 that reduces the set of colors in each unit-segment from at most six to at most three [8], and hence, at the end of this part, the processes of the unit-segment identified by $s t_{i}$ have obtained a proper color within their unit-segment. Moreover, if the process is internal to its unit-segment, it will have obtained its final color (after $\log ^{*} n+3$ rounds).

Message management. Let us observe that, as not all processes start at the same round, it is possible that, while executing a round of the synchronous algorithm of Figure 2 , a process $p_{i}$ receives a message $\operatorname{COLOR}(0, s t,-)$ with $s t \neq$ $s t_{i}$ from its predecessor, or messages $\operatorname{CoLoR}(j,-)$ (where $j \in\{1,2,3\}$, sent in Parts 3 or 4) from one or both of its neighbors. To simplify and make clearer the presentation, the reception of these messages is not indicated in Figure 2. It is implicitly assumed that, when they are received during a synchronous round, these messages are saved in the local memory of $p_{i}$ (so that they can be processed later, if needed, at lines 25-28 and line 39 of Figure 3).

Moreover, a process $p_{i}$ learns the starting round of pred $_{i}$ (resp., next $t_{i}$ ) when it receives for the first time a message $\operatorname{CoLOR}(0, s t,-)$ from $\operatorname{pred}_{i}$ (resp. next $_{i}$ ). To not overload the presentation, this is left implicit in the description of the algorithm.

Part 3 and Part 4. These parts are described in Figure 3. If $p_{i}$ is a left end, or a right end, or both, of a unit-segment ${ }^{6}$, its color at the end of Part 2 is not necessarily its final color, because Part 1 and Part 2 color different unit-segments independently from each other. Hence, it is possible for two contiguous unit-segments to be such that the left end of one, say $p_{i}$, and the right end of the other, say $p_{j}$, have color $_{i}=$ color $_{j}$.

The aim of Part 3 and Part 4 is to resolve these coloring conflicts. To this end, each process $p_{i}$ manages six local variables, denoted $\operatorname{color}_{i}[j, n b g]$, where $j \in\{1,2,3\}$ and $n b g \in\left\{\right.$ pred $_{i}$, next $\left._{i}\right\}$. They are initialized to -1 (no color).

Solving the conflict between neighbors belonging to contiguous unit-segments. A natural idea to solve a coloring conflict between two neighbor processes belonging to different unit-segments, consists in giving "priority" to the unitsegment whose starting time is the first.

Let $s t_{i}\left[\right.$ pred $\left._{i}\right]$ (resp., $s t_{i}\left[\right.$ next $\left._{i}\right]$ ) be the knowledge of $p_{i}$ on the starting time of its left (resp., right) neighbor. If pred $_{i}$ has not yet started let $s_{i}\left[\right.$ pred $\left._{i}\right]=$ $+\infty$ (and similarly for $n e x t_{i}$ ). Thanks to this information, $p_{i}$ knows if it is at the left (resp., right) end of a unit-segment: this is the case if $s t_{i} \neq s t_{i}\left[\right.$ pred $\left._{i}\right]$ (resp., if $s t_{i} \neq s t_{i}\left[\right.$ next $\left._{i}\right]$ ). Moreover, if $p_{i}$ is a left (resp., right) end of a unitsegment, it knows that it has not priority if $s t_{i}>s t_{i}\left[\right.$ pred $\left._{i}\right]$ (resp., $s t_{i}>$ $s t_{i}\left[\right.$ next $\left.\left._{i}\right]\right)$. If such cases, $p_{i}$ may be required to change its color to ensure it differs from the color of its neighbor belonging to the priority contiguous unit-segment.

\footnotetext{
${ }^{6}$ If $p_{i}$ is both a left end and a right end of a unit-segment, it forms its own unit-segment.
} 
In the following parts of the algorithm, each process $p_{i}$ uses local variables denoted color $_{i}[j, n b g]$, where $j \in\{1,2,3\}$ and $n b g \in\left\{\right.$ pred $_{i}$, next $\left._{i}\right\}$.

These variables are initialized to -1 (no color) and updated when $p_{i}$ receives a message $\operatorname{Color}(j,-)$ from pred $_{i}$ or next $_{i}$. Due to the fact that the processes do not start the algorithm at the same round, process $p_{i}$ may have received messages $\operatorname{COLOR}(j,-)$ during previous synchronous rounds. $==$ Part $3:$ color $_{i}$ can be changed only if $p_{i}$ is the left end of its unit-segment

(29) if $\left(s t_{i}>s t_{i}\left[\right.\right.$ pred $\left.\left._{i}\right]\right)$ then $\% p_{i}$ has not priority

(30) $\operatorname{case}\left(s t_{i}=s t_{i}\left[\right.\right.$ next $\left.\left._{i}\right]\right)$ then

$\quad\left(s t_{i}>s t_{i}\left[\right.\right.$ next $\left.\left._{i}\right]\right)$ then $\{0,1,2\} \backslash\left\{\right.$ color $_{i}\left[2\right.$, pred $\left._{i}\right]$, color $_{i}\left[1\right.$, next $\left.\left._{i}\right]\right\}$

(33) end case

$$
\text { color }_{i} \leftarrow \text { a color in }\{0,1,2\} \backslash\left\{\text { color }_{i}\left[2, \text { pred }_{i}\right], \text { color }_{i}\left[2, \text { next }_{i}\right]\right\}
$$

(35)end synchronous round;

$==$ Part $4:$ color $_{i}$ can be changed only if $p_{i}$ is the right end of its unit-segment

(36) when $r=\left(s t_{i}-1\right)+\log ^{*} n+5$ do

(37)begin synchronous round

(38) send $\operatorname{COLOR}\left(2\right.$, color $\left._{i}\right)$ to pred $_{i}$ and next $_{i}$;

(39) same statements as in lines 25-28;

(40) if $\left(s t_{i}>s t_{i}\left[\right.\right.$ next $\left.\left._{i}\right]\right)$ then $\% p_{i}$ has not priority

(41) $\quad$ case $\left(s t_{i}=s t_{i}\left[\right.\right.$ pred $\left.\left._{i}\right]\right)$ then color $_{i} \leftarrow$ a color in $\{0,1,2\} \backslash\left\{\right.$ color $_{i}\left[2\right.$, pred $\left._{i}\right]$, color $_{i}\left[3\right.$, next $\left.\left._{i}\right]\right\}$
$\left(\right.$ st $_{i}>s_{i}\left[\right.$ pred $\left.\left._{i}\right]\right)$ then color $_{i} \leftarrow$ a color in $\{0,1,2\} \backslash\left\{\operatorname{color}_{i}\left[3\right.\right.$, pred $\left._{i}\right]$, color $_{i}\left[3\right.$, next $\left.\left._{i}\right]\right\}$

$\left(s_{i}<t_{i}\left[\right.\right.$ pred $\left.\left._{i}\right]\right)$ then color $_{i} \leftarrow$ a color in $\{0,1,2\} \backslash\left\{\right.$ color $_{i}\left[3\right.$, next $\left.\left._{i}\right]\right\}$

(44) end case

(45) end if

(46)end synchronous round;

$==$ Additional round to inform the neighbors that will start later

(47) when $r=\left(s_{i}-1\right)+\log ^{*} n+6$ do send $\operatorname{COLOR}\left(3\right.$, color $\left._{i}\right)$ to pred $_{i}$ and next $_{i}$; (48)return $\left(\right.$ color $\left._{i}\right)$.

Fig. 3 Part 3 and Part 4 of AST-CV (code for $p_{i}$ )

The tricky cases are the ones of the unit-segments composed of either a single process $p$ or two processes $p_{a}$ and $p_{b}$. This is because, in these cases, it can be required that $p$ (possibly twice, once as right end, and once as left end of its unit-segment), or once $p_{a}$ and once $p_{b}$ (in the case of a 2-process unitsegment), be forced to change the color they obtained at the end of Part 2, to obtain a final color consistent with respect to their neighbors in contiguous unit-segments. To prevent inconsistencies from occurring, it is required that (in addition to the previous priority rule) (a) first a left end process of a unit- 
segment modifies its color with respect to its predecessor neighbor (which belongs to its left unit-segment), and (b) only then a right end process of a unit-segment modifies its color if needed (this specific order is immaterial; the other order -first right, then left- would be equally fine).

Summary of the conflict resolution rules. Let us consider a process $p_{i}$.

- If $p_{i}$ is inside a unit-segment (i.e., $s t_{i}=s t_{i}\left[\right.$ pred $\left._{i}\right]=s t_{i}\left[\right.$ next $\left._{i}\right]$ ), or is the left end of a unit-segment and pred $_{i}$ began after it (i.e., $s t_{i}<s t_{i}\left[\right.$ pred $\left._{i}\right]$ ), or is the right end of a unit-segment and next $t_{i}$ began after it (i.e., $s t_{i}<$ $s t_{i}\left[\right.$ next $\left.\left._{i}\right]\right)$, then the color it obtained at the end of Part 2 is its final color.

- If $p_{i}$ is the left end of a unit-segment and pred $_{i}$ began before $p_{i}$ (i.e., $s t_{i}>s t_{i}\left[\right.$ pred $\left.\left._{i}\right]\right)$, then $p_{i}$ may be forced to change its color. This is done in Part 3. The color $p_{i}$ obtains at the end of Part 3 will be its final color, if it is not also the right end of its unit-segment and next $t_{i}$ began before it (i.e., $s t_{i}>s t_{i}\left[\right.$ next $\left.\left._{i}\right]\right)$.

- This case is similar to the previous one. If $p_{i}$ is the right end of a unitsegment and $n e x t_{i}$ began before it (i.e., $s t_{i}>s t_{i}\left[\right.$ next $\left.\left._{i}\right]\right), p_{i}$ may be forced to change its color to have a final color different from the one of next $t_{i}$. This is done in Part 4.

Recall that a process that is neither the left end, nor the right end of a unit-segment obtains its final color in Part 2. It follows that, during Part 3 and Part 4, such a process only needs to execute the sending of messages $\operatorname{COLOR}(j,-), j \in\{1,2,3\}$ it lines 24,38 , and 47 (the other statements cannot change its color).

Part 3. This part is composed of a single round (lines 22-35). A process $p_{i}$ sends first to its neighbors a message $\operatorname{COLOR}(1, c)$ carrying the color $c$ it has obtained at the end of Part 2. Then, according to the messages it received from them up to the current round, $p_{i}$ updates its local variables color $i\left[j\right.$, pred $\left._{i}\right]$ and color $_{i}\left[j\right.$, next $\left._{i}\right]$ (lines 25-28).

Part 4. This part, composed of a single round (lines 36-46), is similar to the previous one. Due to the predicate of line 40, the lines 41-44 are executed only if $p_{i}$ is the right end of its unit segment. Their meaning is similar to the one of lines 30-33. Finally, $p_{i}$ sends (line 47) to its two neighbors the message $\operatorname{COLOR}\left(3\right.$, color $\left._{i}\right)$ to inform them of its last color, in case it was modified in Part 4.

An example of an execution of $A S T-C V$. Let us consider that $p_{\ell}, p_{a}, p_{b}$, and $p_{r}$ are four consecutive processes such that (i) $s t_{\ell}=10$, and $p_{\ell}$ obtained the final color 1 , (ii) $s t_{r}=12$, and $p_{r}$ obtained the final color 2 , and (iii) $p_{a}$ and $p_{b}$ starts the algorithm at time 15 . Hence, $p_{a}$ and $p_{b}$ define a unit-segment, whose starting time is greater than the one of both $p_{\ell}$ and $p_{r}$. Hence, the unit segment composed of $p_{a}$ and $p_{b}$ has not priority with respect to its two contiguous unit-segments. 
Let us suppose that after having executed Part 1 and Part 2, $p_{a}$ obtains the color 1 , while $p_{b}$ obtains the color 2 , i.e., each obtains a color different from its neighbor in the same unit-segment, but this color is the same as the one of its other neighbor (which belongs to a contiguous "older" unit-segment).

As $p_{a}$ is the left end of its unit-segment and started after $\operatorname{pred}_{a}\left(=p_{\ell}\right)$, it received the message $\operatorname{Color}(2,1)$ from $p_{\ell}$ (line 26 ), and consequently obtains color $_{a}\left[2\right.$, pred $\left._{a}\right]=1$. Moreover, as $p_{a}$ is in the same unit-segment as $p_{b}$, it receives the message $\operatorname{CoLOR}(1,2)$ from $p_{b}$ and obtains color $_{a}\left[1\right.$, next $\left._{a}\right]=2$ (line 27). Then process $p_{a}$ executes lines 29-30, and obtains the color 0 , which happens because

$$
\{0,1,2\} \backslash\left\{\text { color }_{a}\left[2, \text { pred }_{a}\right], \text { color }_{a}\left[1, \text { next }_{a}\right]\right\}=\{0,1,2\} \backslash\{1,2\}=\{0\} .
$$

As $s t_{b}=s t_{a}, p_{b}$ does not execute lines 30-33, but received the message $\operatorname{COLOR}(2,0)$ from $p_{a}$ at line 39 , and we have consequently color $b\left[2\right.$, pred $\left._{b}\right]=0$. It also received $\operatorname{CoLOR}(3,2)$ from $p_{r}$ (line 39), and we have $\operatorname{color}_{b}\left[3\right.$, next $\left._{b}\right]=2$. Process $p_{b}$ then executes lines 40-41, and its final colors is 1 as

$$
\{0,1,2\} \backslash\left\{\operatorname{color}_{b}\left[2, \text { pred }_{b}\right], \text { color }_{b}\left[3, \text { next }_{b}\right]\right\}=\{0,1,2\} \backslash\{0,2\}=\{1\} .
$$

It follows that the final colors of the sequence of the four processes $p_{\ell}, p_{a}$, $p_{b}$, and $p_{r}$ is $1,0,1,2$.

\subsection{Properties of the algorithm}

AST-CV inherits the two most important properties from CV86: locality and determinism.

- The locality property of CV86 states that a process obtains its final color by $\log ^{*} n+3$ rounds. In AST-CV, it obtains it $\log ^{*} n+6$ rounds after its starting round.

- In CV86, the determinism property states that the final color of a process depends only of the identities of the consecutive processes which are its $\log ^{*} n+3$ predecessors on the ring. In AST-CV, its final color depends only of the starting times and the identities of the consecutive processes which are its $\log ^{*} n+6$ predecessors on the ring.

\subsection{Proof of the algorithm}

Definition 1 The final color of a process is the color it returns at line 48 .

Lemma 1 Let $p_{i}$ be a process which wakes up at time st $_{i}$. After $p_{i}$ has executed the round $\left(s_{i}-1\right)+\log ^{*} n+3$ (Part 1 of Figure 2$)$, no two neighbors of its unitsegment have the same color. Moreover, their colors are in the set $\{0,1,2\}$. 
Proof The proof follows from the observation that, when considering the processes of a unit-segment, Part 1 and Part 2 of Figure 2 boils down to CV86, from which the lemma follows.

Lemma 2 Let $p_{i}$ be a process that wakes up. If $p_{i}$ is neither the left end, nor the right end, of its unit-segment, its final color is the color it obtains at the end of Part 2.

Proof If $p_{i}$ is neither the left end nor the right end of its unit-segment we have $s t_{i}=s t_{i}\left[\right.$ pred $\left._{i}\right]=s t_{i}\left[\right.$ next $\left._{i}\right]$. The lemma follows then directly from the predicates of lines 29 and 40 .

$\square$ Lemma 2

Lemma 3 If $p_{i}$ wakes up, its final color belongs to $\{0,1,2\}$.

Proof The proof follows from Lemma 1 and the fact, whatever the lines 30-32 and 41-43 executed by a process $p_{i}$ (if some are ever executed), any of them restricts the new color to belong to the set $\{0,1,2\}$.

$\square_{\text {Lemma } 3}$

Lemma 4 Let us assume that both $p_{i}$ and $p_{j}$ wake up, where $p_{j}$ is $p_{\text {next }}$. If $p_{i}$ and $p_{j}$ belong to the same unit-segment $\left(s t_{j}=s t_{i}\right)$ their final colors are different.

Proof The proof is a case analysis. There are four cases, namely:

Case (a): $p_{i}$ is not the left end and $p_{j}$ is not the right end of their unit-segment, Case (b): $p_{i}$ is not the left end and $p_{j}$ is the right end of their unit-segment, Case (c): $p_{i}$ is the left end and $p_{j}$ is not the right end of their unit-segment, Case $(\mathrm{d}): p_{i}$ is the left end and $p_{j}$ is the right end of their unit-segment.

Case (a): $p_{i}$ is not the left end and $p_{j}$ is not the right end of their unit segment. In this case, it follows from Lemma 1 and Lemma 2 that the final color of $p_{i}$ and the final color of $p_{j}$ are different.

Case (b): $p_{i}$ is not the left end and $p_{j}$ is the right end of their unit-segment. Then, by Lemma 2, the final color of $p_{i}$ is the value of color $r_{i}$ at the end of Part 2 (round $\left.\left(s t_{i}-1\right)+\log ^{*} n+3\right)$. By the algorithm, $p_{j}$ does not change its color at round $\left(s t_{i}-1\right)+\log ^{*} n+4$ (predicate of line 29 where $s t_{i}=s t_{i}\left[\right.$ pred $\left.\left._{i}\right]\right)$, but may change it during round $\left(s t_{i}-1\right)+\log ^{*} n+5$ (Part 5$)$. There are two sub-cases.

$-s t_{j}<s t_{j}\left[\right.$ next $\left._{j}\right]$. In this case the predicate of line 40 is false, and $p_{j}$ does not modify color $_{j}$. It then follows that both $p_{i}$ and $p_{j}$ keep the color they obtained at the end of Part 2. By Lemma 1, these colors are different.

$-s t_{j}>s t_{j}\left[n e x t_{j}\right]$. In this case, $p_{j}$ executes the update of line 41 , where the color assigned to color $_{j}$ remains different from color $_{i}$ (which was received during a previous round and saved in its local variable color $_{j}\left[2\right.$, pred $\left._{j}\right]$ ). 
Case (c): $p_{i}$ is the left end and $p_{j}$ is not the right end of their unit-segment. By Lemma $2, p_{j}$ does not change its color after Part $2\left(\right.$ round $\left.\left(s t_{i}-1\right)+\log ^{*} n+3\right)$. There are two cases.

$-s t_{i}<s t_{i}\left[\right.$ pred $\left._{i}\right]$. It follows from the predicate of line 29 that $p_{i}$ does not change its color during Part 3. As $s t_{i}=s t_{j}$, the predicate of line 40 is false, and $p_{i}$ does not change its color in Part 4 . It then follow from Lemma 1 that $p_{i}$ and $p_{j}$ have different final colors.

$-s t_{i}>s t_{i}\left[\right.$ pred $\left._{i}\right]$. As $p_{i}$ and $p_{j}$ are in the same unit-segment, $p_{i}$ receives $\operatorname{COLOR}\left(1\right.$, color $\left._{j}\right)$ at line 27 during the round $\left(s t_{i}-1\right)+\log ^{*} n+4$ (Part 3 ), and saves this value in its local variable $\operatorname{color}_{i}\left[1\right.$, next $\left._{i}\right]$. Then, due to the predicates of lines 29 and 30, $p_{i}$ changes its color at line 30 during the round $\left(s t_{i}-1\right)+\log ^{*} n+4$ (Part 3$)$, and this color is different from the final color of $p_{j}$. Finally, as $s t_{i}=s t_{j}$, the predicate of line 40 is not satisfied, and $p_{i}$ does not update color $i$ during the round $\left(s t_{i}-1\right)+\log ^{*} n+5$ (Part $4)$. It then follows from that $p_{i}$ and $p_{j}$ have different final colors.

Case $(\mathrm{d}): p_{i}$ is the left end and $p_{j}$ is the right end of their unit-segment. There are four cases.

$-s t_{i}<s t_{i}\left[\right.$ pred $\left._{i}\right]$ and $s t_{j}<s t_{j}\left[n e x t_{j}\right]$. In this case, $p_{i}$ and $p_{j}$ do not change their color after round $\left(s t_{i}-1\right)+\log ^{*} n+3$. Hence, by Lemma 1 , they will have different final colors.

$-s t_{i}<s t_{i}\left[\right.$ pred $\left._{i}\right]$ and $s t_{j}>s t_{j}\left[\right.$ next $\left._{j}\right]$. In this case, when evaluated by $p_{i}$, the predicates of lines 29 and 40 (we have then $s t_{i}=s t_{i}\left[n e x t_{i}\right]=s t_{j}$ ) are false. Hence, $p_{i}$ does not change its color after round $\left(s t_{i}-1\right)+\log ^{*} n+3$. This case is similar to the second sub-case of Case (b).

$-s t_{i}>s t_{i}\left[\right.$ pred $\left._{i}\right]$ and $s t_{j}<s t_{j}\left[n e x t_{j}\right]$. In this case $p_{j}$ does not change its color after Part 2 (round $\left.\left(s t_{j}-1\right)+\log ^{*} n+3\right)$. This case is similar to the second sub-case of Case (c).

$-s t_{i}>s t_{i}\left[\right.$ pred $\left._{i}\right]$ and $s t_{j}>s t_{j}\left[\right.$ next $\left._{j}\right]$. Due to the predicates of lines 29 and $30, p_{i}$ changes its color at line 30 during round $\left(s t_{i}-1\right)+\log ^{*} n+4($ Part 3). Moreover, as $s t_{i}=s t_{j}$, it does not change its color in Part 4 . Hence, its final color is the one obtained at line 30 . Differently, as $s t_{j}>s t_{j}\left[n e x t_{j}\right]$ and $s t_{j}=s t_{i}, p_{j}$ updates its color at line 41 during round $\left(s t_{j}-1\right)+\log ^{*} n+$ 5 (Part 4), where it obtains a color different from color $_{i}$ (final color of $p_{i}$ received at line 38 and saved in $p_{j}$ 's local variable color $j\left[2\right.$, pred $\left.\left._{j}\right]\right)$. It follows that $p_{i}$ and $p_{j}$ have different final colors.

Lemma 5 Let us assume that both $p_{i}$ and $p_{j}$ wake up, where $p_{j}$ is $p_{\text {next }}$. If $p_{i}$ and $p_{j}$ are not in the same unit-segment and $s t_{i}>s t_{j}$, their final colors are different.

Proof The processes $p_{i}$ and $p_{j}$ are neighbors but belong to different unitsegments. As $s t_{j}<s t_{i}$ and all processes gets their final color after the same constant number of round after they wake up, $p_{j}$ gets its final color before $p_{i}$. 
The proof considers the following two possible cases: Case (a): $p_{i}$ is not a left end of its unit segment, and Case (b) $p_{i}$ is a left end of its unit segment.

Case (a): $p_{i}$ is not a left end of its unit segment. In this case, it follows from the predicate of line 29 that $p_{i}$ does not change its color during Part 3 , and from the predicates of lines 40 and 41 (Part 4), that $p_{i}$ updates its color at line 41 . As $p_{j}$ woke up before $p_{i}, p_{i}$ received the message COLOR $(3, \mathrm{col})$ sent at line 47 by $p_{j}$ during its round $\left(s t_{j}-1\right)+\log ^{*} n+6$. This message was received by $p_{i}$ at the latest while it executes its round $\left(s t_{i}-1\right) i+\log ^{*} n+5$. Moreover, $\mathrm{col}$ is then the final color of $p_{j}$. It follows that, when it executes its round $\left(s t_{i}-1\right)+\log ^{*} n+5, p_{i}$ is such that $\operatorname{color}_{i}\left[3\right.$, next $\left._{i}\right]=$ col. Consequently, at line $41, p_{i}$ adopts a final color different from the final color of $p_{j}$.

Case (b): $p_{i}$ is a left end of its unit segment. We consider two sub-cases.

$-s t_{i}<s t_{i}\left[\right.$ pred $\left._{i}\right]$. In this case, it follows from the predicate of line 29 that $p_{i}$ does not change its color during Part 3. Differently, due to the predicates of lines 40 and 43, it updates color $_{i}$ at line 43. Moreover, as $s t_{i}>s t_{j}, p_{i}$ received from $p_{j}$ the message $\operatorname{COLOR}(3, \mathrm{col}$ ) (where $\mathrm{col}$ is the final color of $\left.p_{j}\right)$ at a round $\leq\left(s t_{i}-1\right)+\log ^{*} n+5$, and saved col in color $_{i}\left[3\right.$, next $\left._{i}\right]$. It then follows that, when $p_{i}$ executes line 43 , it assigns to color $r_{i}$ a value different from the final color of $p_{j}$.

$-s t_{i}>s t_{i}\left[\right.$ pred $\left._{i}\right]$. In this case, it follows from the predicates of lines 29 and 31 that $p_{i}$ updates its color at line 31 (Part 3), and from the predicates of lines 40 and 42 that $p_{i}$ updates again its color at line 42 (Part 4 ). As $p_{j}$ woke up before $p_{i}, p_{i}$ received the message $\operatorname{CoLOR}(3, \mathrm{col})$ from $p_{j}$ before (or at) round $\left(s t_{i}-1\right)+\log ^{*} n+5$ (Part 4$)$, and col is the final color of $p_{j}$. It follows that, when $p_{i}$ updates its color at line 42 , we have $\operatorname{color}_{i}\left[3\right.$, next $\left._{i}\right]=$ col. Consequently, the final color of $p_{i}$ is different from the final color of its neighbor $p_{j}$.

Lemma 6 Let us assume that both $p_{i}$ and $p_{j}$ wake up, where $p_{j}$ is $p_{\text {next }}$. If $p_{i}$ and $p_{j}$ are not in the same unit-segment and $s t_{j}>s t_{i}$, their final colors are different.

Proof By assumption, $p_{i}$ and $p_{j}$ are neighbors, but belong to different unitsegments. As $s t_{j}>s t_{i}$ and all processes execute the same number of rounds after they woke up $\left(\log ^{*} n+6\right), p_{i}$ returns its final color (line 48) before $p_{j}$. As for Lemma 4, the proof of the lemma considers four cases, namely Case (a): $p_{i}$ is not the left end of its unit-segment and $p_{j}$ is not the right end of its unit-segment,

Case (b): $p_{i}$ is not the left end of its unit-segment and $p_{j}$ is the right end of its unit-segment,

Case (c): $p_{i}$ is the left end of its unit-segment and $p_{j}$ is not the right end of its unit-segment, 
Case (d): $p_{i}$ is the left end of its unit-segment and $p_{j}$ is the right end of its unit-segment.

Case (a): $p_{i}$ is not the left end of its unit-segment and $p_{j}$ is not the right end of its unit segment. As $p_{i}$ is not the left end of its unit segment, it follows from the predicate of line 29 that it does not update its color in Part 3. As $s t_{i}<s t_{j}=s t_{i}\left[\right.$ next $\left._{i}\right]$, it follows from the predicate of line 40 that $p_{i}$ does not update its color in Part 4. Hence, $p_{i}$ obtained its final color at the end of Part 2.

As far as $p_{j}$ is concerned, we have the following. As $s t_{j}>s t_{i}$ and $p_{j}$ is not the right end of its unit-segment, the predicates of lines 29 and 30 direct $p_{j}$ to update its color at line 30 (Part 3 ). Moreover, as $p_{j}$ is not the right end of its unit-segment, the predicate of line 40 is not satisfied and $p_{j}$ does not change its color in Part 4.

As $p_{i}$ woke up before $p_{j}, p_{j}$ received the message $\operatorname{COLOR}(2, \mathrm{col})$ from $p_{i}$ at a round $\leq\left(s t_{j}-1\right)+\log ^{*} n+4$, and $c o l$ is the final color of $p_{i}$. It follows that when $p_{j}$ executes line 30 , it assigns to color ${ }_{j}$ a color different from the final color of $p_{i}$.

Case (b): $p_{i}$ is not the left end of its unit-segment and $p_{j}$ is the right end of its unit-segment. As $p_{i}$ is not the left end of its unit-segment and $s t_{i}<s t_{j}$, it follows that the predicate of line 29 is not satisfied when evaluated by $p_{i}$. Similarly, as $s t_{i}<s t_{i}\left[n e x t_{i}\right]=s t_{j}$, the predicate of line 40 is not satisfied either. Consequently, $p_{i}$ does not modify its color in Part 3 or Part 4 . Let $c l \_i$ be this color.

As $p_{i}$ wakes up before $p_{j}, p_{j}$ has received the message COLOR $\left(2, c l \_i\right)$ sent by $p_{i}$ at the latest during its round $\left(s t_{j}-1\right)+\log ^{*} n+4$ (Part 3$)$. Hence, at the end of round $\left(s t_{j}-1\right)+\log ^{*} n+4$, color $_{j}\left[2\right.$, pred $\left._{j}\right]=c l \_i$. Moreover, $p_{j}$ received the message COLOR $\left(3, c l \_i\right)$ at the latest during its round $\left(s t_{j}-1\right)+\log ^{*} n+5$, and saved it in color $_{j}\left[3\right.$, pred $\left._{j}\right]=c l_{-} i$. It then follows that, whatever the update of color ${ }_{j}$ done by $p_{j}$ at any line of Part 3 (lines 30-32) or Part 4 (lines 41-43), the final color of $p_{j}$ will be different from the final color of $p_{i}$.

Case (c): $p_{i}$ is the left end of its unit-segment and $p_{j}$ is not the right end of its unit-segment.

As $p_{i}$ is the left end of its unit-segment, it may be forced to update its color (at line 32 because $s t_{j}>s t_{i}$ ) if the predicate of line 29 is satisfied (Part 3). But as $s t_{j}>s t_{i}$, the predicate of line 40 cannot be satisfied (Part 4 ). Hence, both the messages COLOR $\left(2, c l \_i\right)$ and COLOR $\left(3, c l_{-} i\right)$ sent by $p_{i}$ at lines 38 and 47 carry its final color.

As $s t_{j}>s t_{i}, p_{j}$ received $\operatorname{COLOR}\left(2, c l_{-} i\right)$ at the latest during its round $\left(s t_{j}-1\right)+\log ^{*} n+4$, and COLOR $(3, c l-i)$ at the latest during its round $\left(s t_{j}-\right.$ 1) $+\log ^{*} n+5$. It follows that, whatever the update of color $_{j}$ done by $p_{j}$ when it executes Part 3 or Part 4, its final color will be different from $c l \_i$. 
Case (d): $p_{i}$ is the left end of its unit-segment and $p_{j}$ is the right end of its unit-segment.

As indicated in the previous case, $p_{i}$ (left end of its unit-segment) may change its color due the predicates of lines 29 and 32 when it executes its round $\left(s t_{i}-1\right)+\log ^{*} n+4$ (Part 3$)$, but (as $\left.s t_{i}<s t_{j}\right)$ it will not change it in Part 4. We consider two cases. Let $c l_{-} i$ be the final color of $p_{i}$.

$-s t_{j}>s t_{j}\left[n e x t_{j}\right]$. In this case, As $s t_{j}>s t_{i}$ the predicate of line 29 is satisfied, and $p_{j}$ updates its color at line 31 when it executes its round $\left(s t_{j}-1\right)+\log ^{*} n+4$ (Part 3). Similarly, as $s t_{j}>s t_{j}\left[n e x t_{j}\right], p_{j}$ updates its color at line 42 when it executes its round $\left(s t_{j}-1\right)+\log ^{*} n+4$ (Part 4). As $p_{j}$ woke up after $p_{i}$, it received $\operatorname{COLOR}\left(2, c l_{-} i\right)$ from $p_{i}$ at the latest when it executes its round $\left(s t_{j}-1\right)+\log ^{*} n+4$ (Part 3$)$, and received COLOR $\left(3, c l \_i\right)$ at the latest when it executes its round $\left(s t_{j}-1\right)+\log ^{*} n+5$ (Part 4). It follows that, whatever (if any) an update of color $_{j}$ done at any of the lines 30-32 and 41-43, the final color of $p_{j}$ will be different from the one of $p_{i}$.

- $s t_{j}<s t_{j}\left[\right.$ next $\left._{j}\right]$. In this case, $p_{j}$ may update its color at line 32 while executing its round $s t_{j}+\log ^{*} n+4$ (Part 3$)$. As $s t_{i}<s t_{j}, p_{j}$ receives the message $\operatorname{COLOR}\left(2, c l_{-} i\right)$ from $p_{i}$ at the latest during its round $\left(s t_{j}-1\right)+$ $\log ^{*} n+4\left(\right.$ cl $_{-} i$ is the final color of $\left.p_{i}\right)$, and consequently color $_{j}\left[2\right.$, pred $\left._{j}\right]=$ $c l \_i$ at round $\left(s t_{j}-1\right)+\log ^{*} n+4$. Hence, when it executes line $32, p_{j}$ updates color $_{j}$ to a color different from $c l_{-} i$. Let us finally observe that, as $s t_{j}<s t_{j}\left[\right.$ next $\left._{j}\right]$, the predicate of line 40 (Part 4 ) is not satisfied, and consequently $p_{j}$ does not modify color ${ }_{j}$ at lines $41-43$, which completes the proof of the lemma.

$\square_{\text {Lemma } 6}$

The following theorem showing the correctness of algorithm AST-CV directly follows from Lemmas 3, 4, 5 and 6 .

Theorem 1 If $p_{i}$ and $p_{j}$ wake up and are neighbors, their final colors are different and in the set $\{0,1,2\}$.

\section{From Asynchronous Starting Times to Wait-Freedom}

This section presents the WLC (Wait-free Local Coloring) algorithm for the $\mathcal{D E C O U P} \mathcal{L} \mathcal{E D}$ model described in Section 2, which 3-colors the processes of a ring. This algorithm consists of two consecutive stages executed independently by each process $p_{i}$. The first stage is a communication stage during which $p_{i}$, whatever its starting time, obtains enough information to execute its second stage, which consists of a communication-free computation.

The following solvability notion incorporates asynchrony and failures, as needed by the $\mathcal{D E C O U P} \mathcal{L} \mathcal{E}$ model. An algorithm wait-free solves $m$-coloring if for each of its executions: (1) Validity. The final color of any process is in $\{0, \ldots, m-1\}$. (2) Agreement. The final colors of any two neighbor nodes in 
the graph are different. (3) Termination. All processes that take an infinite number of steps decide a final color.

5.1 On the communication side

A ring structure for the synchronous communication network. The neighbors of a node $n d_{i}$ (or process $p_{i}$ with a slight abuse of language) are denoted as before, pred $_{i}$ and next . $_{\text {. }}$

On the side of the communication nodes. While each input buffer $i n_{i}$ is initially empty, each output buffer out $t_{i}$ is initialized to $\langle i,+\infty, \perp\rangle$. When a process starts its participation in the algorithm, it writes the pair $\left\langle i, s t_{i}, i d_{i}\right\rangle$ in out $_{i}$, where $s t_{i}$ is its starting time (as defined by the current tick of the clock governing the progress of the underlying communication component), and $i d_{i}$ is its identity.

As already described, at every clock tick (underlying communication step), $n d_{i}$ first receives two messages (one from each neighbor), and reads the local buffer out ${ }_{i}$. Then, it packs the content of these two messages and the content of out $_{i}$ (which can be $\langle i,+\infty, \perp\rangle$ if $p_{i}$ has not yet started) into a single message, sends it to its two neighbors, and writes it in $i n_{i}$ (full-information behavior of a node).

\subsection{Wait-free algorithm: first a communication stage}

Let $p_{i}$ be a process that starts the algorithm at time $s t_{i}=t$. As previously indicated, this means that, at time $t$ (clock tick defined by the communication component), $p_{i}$ writes $\left\langle i, t, i d_{i}\right\rangle$ in its output buffer out $t_{i}$. Then $p_{i}$ waits until time $t+\Lambda$ where $\Lambda=\log ^{*} n+5 .\left(^{7}\right)$. At the end of this waiting period, and as far $p_{i}$ is concerned, the "dices are cast". No more physical communication will be necessary. As we are about to see, $p_{i}$ obtained enough information to compute alone its color: the rest of the algorithm executed by $p_{i}$ is purely local (see below). This feature, and the fact that the starting time of a process depends only on it, makes the algorithm wait-free.

It follows from the underlying communication component that, at time $t+$ $\Lambda, p_{i}$ has received information (i.e., a triplet $\left\langle j, s t, i d_{j}\right\rangle$ ) from all the processes at distance at most $\Lambda$ of it. If $s t=t, p_{i}$ knows that $p_{j}$ started the algorithm at the same time as itself. If $s t<t$ (resp., st $>t$ ), $p_{i}$ knows that $p_{j}$ started the algorithm before (resp., after) it. (If $s t=+\infty$-we have then $i d_{j}=\perp$ - and $p_{j}$ is at distance $d$ from it, $p_{i}$ knows that $p_{j}$ did not start the algorithm before the clock tick $t+\Lambda-d$.)

\footnotetext{
7 Being asynchronous, the waiting of $p_{i}$ during an arbitrary long (but finite) period does not modify its allowed behavior.
} 
5.3 Wait-free algorithm: then a local simulation stage of AST-CV

At the end of its waiting period, $p_{i}$ has information (triplets composed of an index, a starting time -possibly $+\infty-$, and a process identity -possibly $\perp-$ ) of all the processes at distance $\Lambda=\log ^{*} n+5$ from it, and also from the processes at distance $k$ that started before $s t_{i}+\Lambda-k$ (each triplet from process $p_{j}$ at distance $k$ was propagated from it to a process $p_{\ell}$ at distance $\Lambda$ from $p_{i}$, and then from $p_{\ell}$ to $p_{i}$ ). More precisely, for each of these processes $p_{j}, p_{i}$ knows whether $p_{j}$ started before it $\left(s t_{j}<s t_{i}\right)$, at the same time as it $\left(s t_{j}=s t_{i}\right)$, or after it $\left(s t_{j}>s t_{i}\right)$.

Simulation of $A S T-C V$ It follows from the previous observation that, after its waiting period, $p_{i}$ has all the inputs (starting times and process identities) needed to simulate AST-CV and compute its final color, be it inside a unitsegment, the left end of a unit-segment, the right end of a unit-segment, or both ends of a unit-segment (a maximal sequence of consecutive processes that start the algorithm at the same time).

More precisely, the purely local simulation by a process $p_{i}$ is a follows. Starting from round $0, p_{i}$ simulates $s t_{i}+\Lambda$ rounds of AST-CV, this simulation involving the processes from which it has the initial information $\left\langle j, s t_{j}, i d_{j}\right\rangle$ and are s.t. $s t_{j} \leq s t_{i}$.

Notice that the crash of a process $p_{j}$ has no impact on the termination and the correctness of the coloring of other processes. This follows from the locality property of AST-CV, and the fact that as soon as a process has obtained a triplet $\left\langle j, s t_{j}, i d_{j}\right\rangle$ (where $s t_{j} \leq s t_{i}$ ), it considers $p_{j}$ as competing for a color, whatever is its behavior after it started participating in the algorithm.

Optimality of WLC When it executes WLC, each process waits during $O\left(\log ^{*} n\right)$ time units, which occurs during the communication phase. This duration is asymptotically optimal as (1) $\Omega\left(\log ^{*} n\right)$ is a lower bound on the number of time units needed to color the nodes of a ring with at most three colors [18] in $\mathcal{L O C} \mathcal{A} \mathcal{L}$, and (2) when there is neither asynchrony nor failures, $\mathcal{D E C O U P} \mathcal{L} \mathcal{E}$ behaves like $\mathcal{L O C} \mathcal{A} \mathcal{L}$.

\section{Conclusion}

The paper proposed a model where communication and processing are decoupled, consisting of asynchronous crash-prone processes that run on top of a

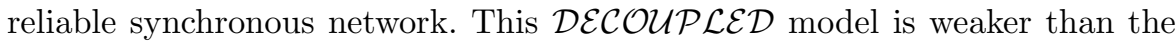
synchronous model (on the process side) and stronger than the asynchronous crash-prone model (on the communication side), while encompassing in a single framework two fundamental issues of distributed computing, locality [18] and wait-freedom [15].

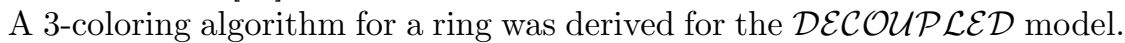
This algorithm uses as a subroutine a generalization of Cole and Vishkin's 
algorithm [8]. A process needs to obtain initial information from processes at distance at most $O\left(\log ^{*} n\right)$ of it. As far as we know, this is the first wait-free local coloring algorithm, which colors a ring with at most three colors.

In contrast to $\mathcal{L O C} \mathcal{A L}$, in the $\mathcal{D E C O U} \mathcal{P} \mathcal{L} \mathcal{D}$ model, after $d$ rounds of communication, a process collects the initial inputs of only a subgraph of its $d$ neighborhood. The paper has shown that, despite this uncertainty, it is possible to combine locality and wait-freedom, as far as 3-coloring is concerned. The keys to this marriage were (a) the decoupling of communication and processing, and (b) the design of a synchronous coloring algorithm (AST-CV), where the processes are reliable, proceed synchronously, but are not required to start at the very same round, which introduces a first type of asynchrony among the processes. As we have seen, the heart of this algorithm lies in the consistent coloring of the border vertices of subgraphs which started at different times (unit segments).

It would be interesting if this methodology applies to other coloring algorithms, or even to other distributed graph problems which are solvable in the $\mathcal{L O C} \mathcal{A} \mathcal{L}$ model. Variants of the $\mathcal{D E C O U} \mathcal{P} \mathcal{L} \mathcal{E}$ model might be interesting to study, e.g., several asynchronous processes running in the same node.

\section{Acknowledgments}

C. Delporte, H. Fauconnier, and M. Raynal, were partially supported by the French ANR project DESCARTES, devoted to abstraction layers in distributed computing. A. Castañeda was supported in part by UNAM PAPIITDGAPA projects IA101015 and IA102417. S. Rajsbaum was supported in part by UNAM PAPIIT-DGAPA project IN107714.

All the authors want to thank INRIA for its support in the context of the INRIA-UNAM "Équipe Associée" LiDiCo (At the Limits of Distributed Computing).

\section{References}

1. Arjomandi E., Fischer M., and Lynch N., Efficiency of Synchronous Versus Asynchronous Distributed Systems. Journal of the ACM, 30(3):449-456 (1983)

2. Awerbuch B., Complexity of network synchronization. JACM, 32(4): 804-823, (1985)

3. Awerbuch B., Patt-Shamir B., Peleg D., and Saks M., Adapting to asynchronous dynamic networks (extended abstract). Proc. 24th ACM symposium on Theory of computing (STOC'92), pp. 557-570 (1992)

4. Barenboim L., Deterministic $(\Delta+1)$-coloring in sublinear (in $\Delta)$ time in static, dynamic, and faulty networks. Journal of the ACM, 63(5), Article 47 (2016)

5. Barenboim L. and Elkin M., Deterministic distributed vertex coloring in polylogarithmic time. Journal of the ACM, 58(5), 23 pages (2011)

6. Barenboim L. and Elkin M., Distributed graph coloring, fundamental and recent developments, Morgan \& Claypool Publishers, 155 pages (2014)

7. Barenboim L., Elkin M., and Kuhn F., Distributed (Delta+1)-coloring in linear (in Delta) time. SIAM Journal of Computing, 43(1):72-95 (2014)

8. Cole R. and Vishkin U., Deterministic coin tossing with applications to optimal parallel list ranking. Information and Control, 70(1):32-53 (1986) 
9. Fischer M.J., Lynch N.A., and Paterson M.S., Impossibility of distributed consensus with one faulty process. Journal of the ACM, 32(2):374-382 (1985)

10. Fraigniaud P., Heinrich M., and Kosowski A., Local conflict coloring. Proc. 57th IEEE Annual Symposium on Foundations of Computer Science, IEEE Computer Society, pp. 625-634 (2016)

11. Fraigniaud P., Gafni E., Rajsbaum S., and Roy M., Automatically adjusting concurrency to the level of synchrony. Proc. 28th Int. Symp. Distributed Computing (DISC'14), Springer LNCS 8784, pp. 1-15 (2014)

12. Fraigniaud P., Korman A., and Peleg D., Towards a complexity theory for local distributed computing. Journal of the ACM, 60(5), Article 35, 16 pages (2013)

13. Garey M.R. and Johnson D.S., Computers and intractability: a guide to the theory of NP-completeness. W.H. Freeman, New York, 340 pages (1979)

14. Goldberg A., Plotkin S, and Shannon G., Parallel symmetry-breaking in sparse graphs. SIAM Journal on Discrete Mathematics, 1(4):434-446 (1988)

15. Herlihy M.P., Wait-free synchronization. ACM Transactions on Programming Languages and Systems, 13(1):124-149 (1991)

16. Keidar I. and Rajsbaum S., On the cost of fault-tolerant consensus when there are no faults: preliminary version. ACM SIGACT News, 32(2):45-63 (2001)

17. Kuhn F., Moscibroda T., and Wattenhofer R., What cannot be computed locally! Proc. 23rd ACM Symposium on Principles of Distributed Computing, ACM Press, pp. 300309 (2004)

18. Linial N., Locality in distributed graph algorithms. SIAM JC, 21(1):193-201 (1992)

19. T. Meincke et al., Globally asynchronous locally synchronous architecture for large highperformance ASICs. Proceedings of the IEEE International Symposium on Circuits and Systems (ISCAS'99), pp. 512-515, vol. 2 (1999)

20. Naor M. and Stockmeyer L., What can be computed locally? SIAM Journal on Computing, 24(6):1259-1277 (1995)

21. Peleg D., Distributed computing, a locally sensitive approach. SIAM Monographs on Discrete Mathematics and Applications, 343 pages, ISBN 0-89871-464-8 (2000)

22. Raynal M., Fault-tolerant agreement in synchronous message-passing systems. Morgan \& Claypool Publishers, 165 pages, ISBN 978-1-60845-525-6 (2010)

23. Raynal M., Communication and agreement abstractions for fault-tolerant asynchronous distributed systems. Morgan \& Claypool Publishers, 251 pages, ISBN 978-1-60845-293-4 (2010)

24. Raynal M., Concurrent programming: algorithms, principles, and foundations. Springer, 530 pages, ISBN 978-3-642-32026-2 (2013)

25. Suomela J., Survey of local algorithms. ACM Computing Surveys, 45(2), Art. 24, 40 pages (2013) 